\title{
Neonatal Progeroid Syndrome
}

National Cancer Institute

\section{Source}

National Cancer Institute. Neonatal Progeroid Syndrome. NCI Thesaurus. Code C121565.

A rare autosomal recessive disorder associated with abnormalities in bone maturation, and lipids and hormone metabolism and characterized by intrauterine growth retardation, failure to thrive, short stature, a progeroid appearance, hypotonia, variable mental impairment, and death in childhood. Its cause is unknown. 